\title{
Continuous measurements of atmospheric water vapour isotopes in
} western Siberia (Kourovka)

\section{Bastrikov et al.}

Correspondence to: V. Bastrikov (v.bastrikov@gmail.com) 


\section{Supplementary material}

\section{Operational time intervals estimation}

We perform a calibration of our Picarro L2130-i analyzer after every six hours of ambient air measurements. Each calibration is made with two reference liquid standards, each standard is continuously measured for $30 \mathrm{~min}$.

Figure $\mathrm{S} 1$ shows an example of $\delta \mathrm{D}$ measurements during a calibration cycle. Calibration intervals (30 minutes) are shown in red with the last 3 minutes shown in blue. On the zoomed fragments it can be seen that representative measurements are established only in the very end of calibration interval. With the larger isotopic difference between the two adjacently measured samples the effect becomes more pronounced. We find it reasonable to calculate the resulting value of each successful calibration over the last three minutes in a steady plateau area.
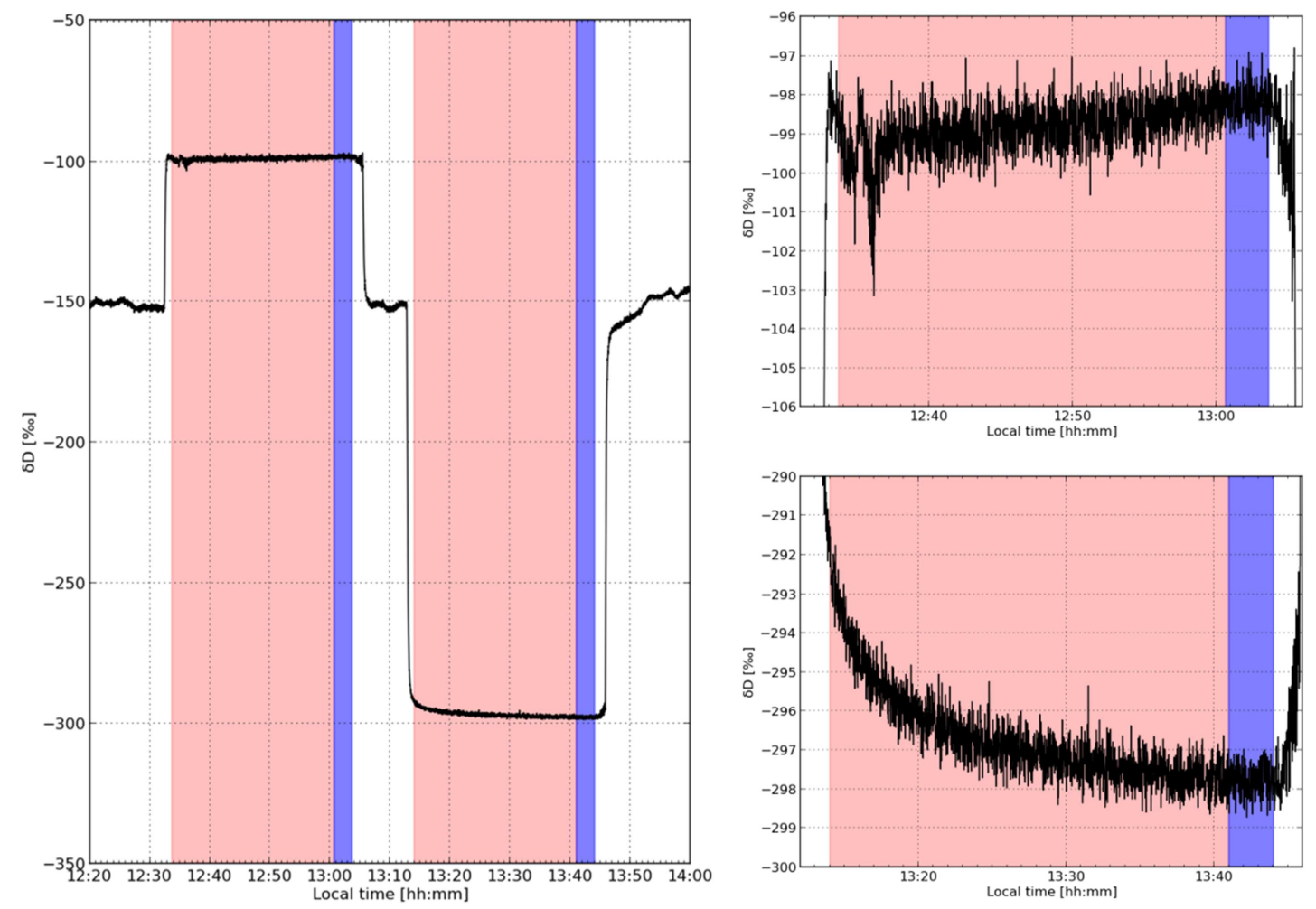

Figure S1. Example of $\delta \mathrm{D}$ measurements during the calibration time-series (left) with zoomed regions (right). Red - calibration period (30 minutes), blue - representative part ( 3 minutes). 
The ambient air measurements performed by the isotope analyzer immediately after calibration are influenced by the residuals of calibration water vapour contained in the system. Figure S2 shows an example for $\delta \mathrm{D}$ measurements during the instrument switching from calibration to ambient air measurements (the first 13 minutes are shown in red). The switching occurs at 08:29 LT, the mean ambient $\delta \mathrm{D}$ value equals to $-134.4 \%$. At 4 minutes after the switching the instrument shows $-138.7 \%$ ( $3.2 \%$ error), at 8 minutes it shows $-136.2 \%$ ( $1.3 \%$ error) and at 12 minutes it shows $-135.1 \%$ o ( $0.5 \%$ error). Such an effect occurs each time the instrument switches between the samples with largely different isotopic composition. We find it appropriate to remove the first 13 minutes of the measurements after each calibration cycle.
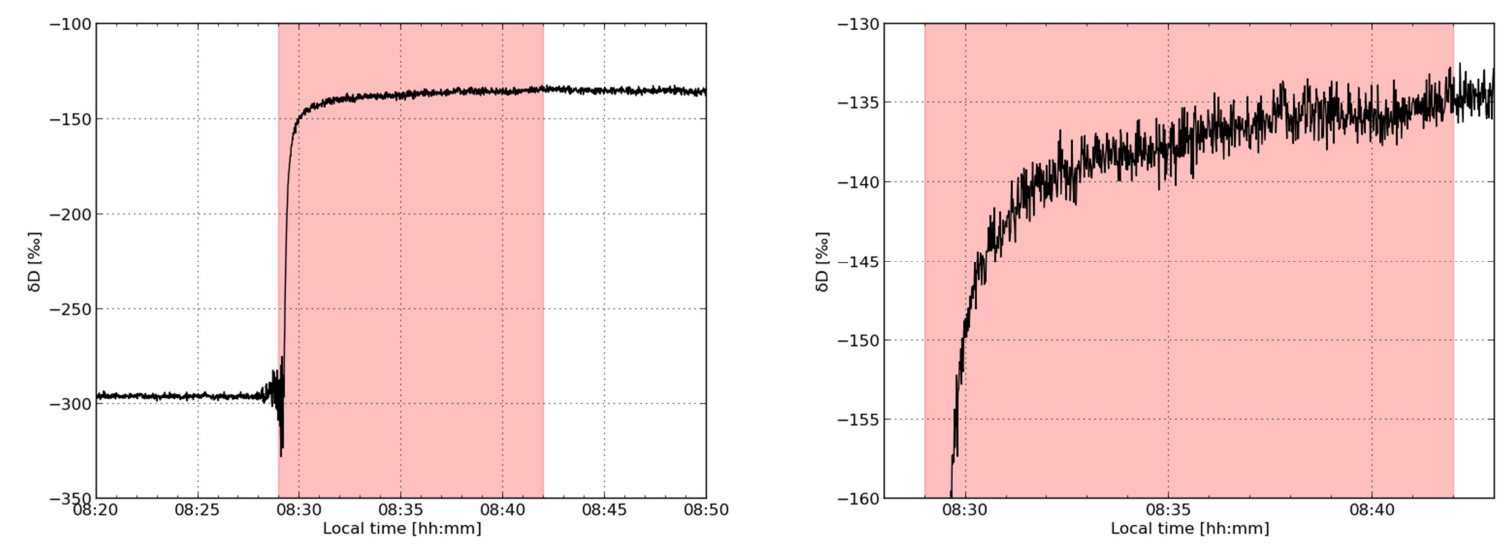

Figure S2. Example of $\delta \mathrm{D}$ measurements during the instrument switching from calibration to ambient air measurements (left) with a zoomed region (right). Red - discarded part of the measurements (13 minutes).

\section{Cluster analysis}

We have performed an analysis of all available meteorological parameters associated with each of the two dominant diurnal cycle patterns (clusters) obtained for deuterium excess (Fig. S3). 
The stacked diurnal cycles for each parameter are shown on the left panels on Fig. S3 with the color indicating the corresponding d-excess cluster (red Cluster 1 with the strong decrease of d-excess during the night, blue - Cluster 2 with the weak decrease of d-excess during the night). Resulting clusters for each parameter are shown on the right panels on Fig. S3 with corresponding standard deviations shown by shading. The yellow bars show the time of sunrise and the dark red bars show the time of sunset. For each humidity cycle (Fig. S3, b) we have additionally subtracted the difference between its mean value and the overall mean value and juxtaposed the resulting centroids in the time interval between 05:00 LT and 06:00 LT (Fig. S3, c). This was done for better representation of the humidity morning burst difference and does not distort the clusters itself. All the other data were computed directly.

Only for the absolute humidity (Fig. S3, c) and wind speed (Fig. S3, d) do we observe a significant difference between the diurnal variations corresponding to the two d-excess clusters. However, we observe indications of slightly different meteorological conditions associated with the two d-excess clusters. For Cluster 1 we observe a more pronounced diurnal cycle in temperature (Fig. S3, e) and relative humidity (Fig. S3, f) and in general slightly higher pressure (Fig. S3, g). Cluster 2 does on the contrary not show as strong diurnal cycle for these parameters and the atmospheric pressure is slightly lower. This indicates that Cluster 1 is associated with more stable atmospheric conditions, which allow the wind speed to become very small during the night creating a stratiform lower boundary.

The wind direction diurnal cycle (Fig. S3, h) appears to be not representative, as the wind speed values are usually quite small for the establishment of an appropriate wind direction signal. 
a)

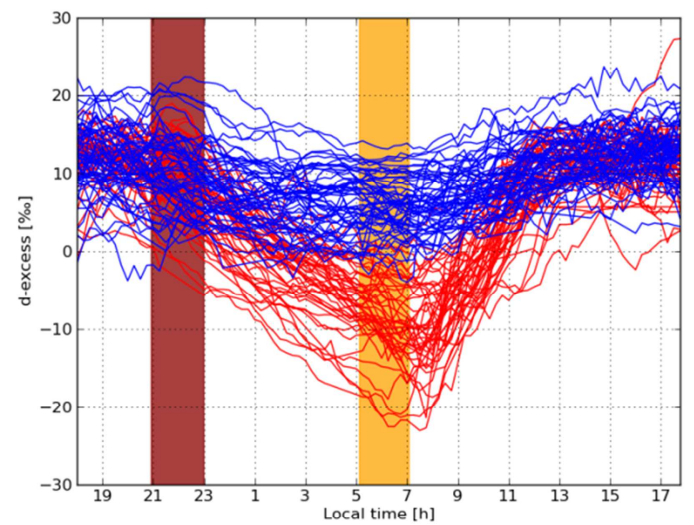

b)

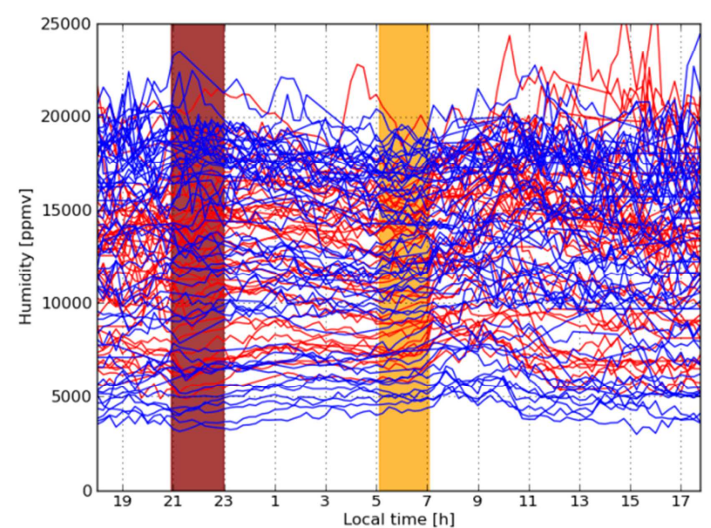

c)

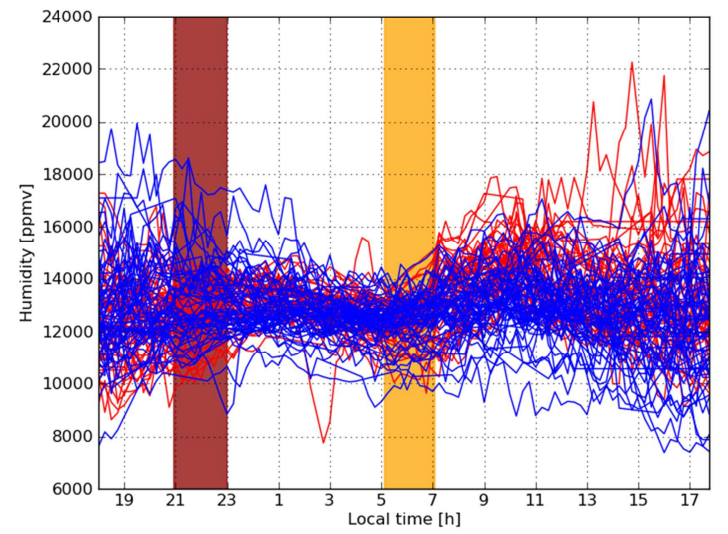

d)

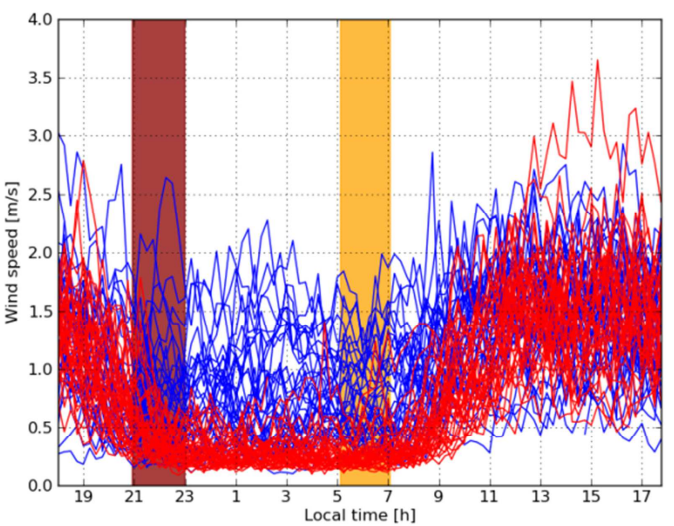

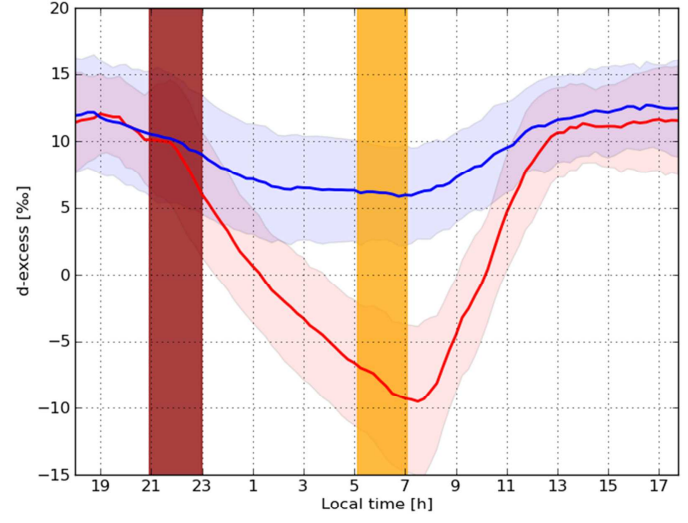
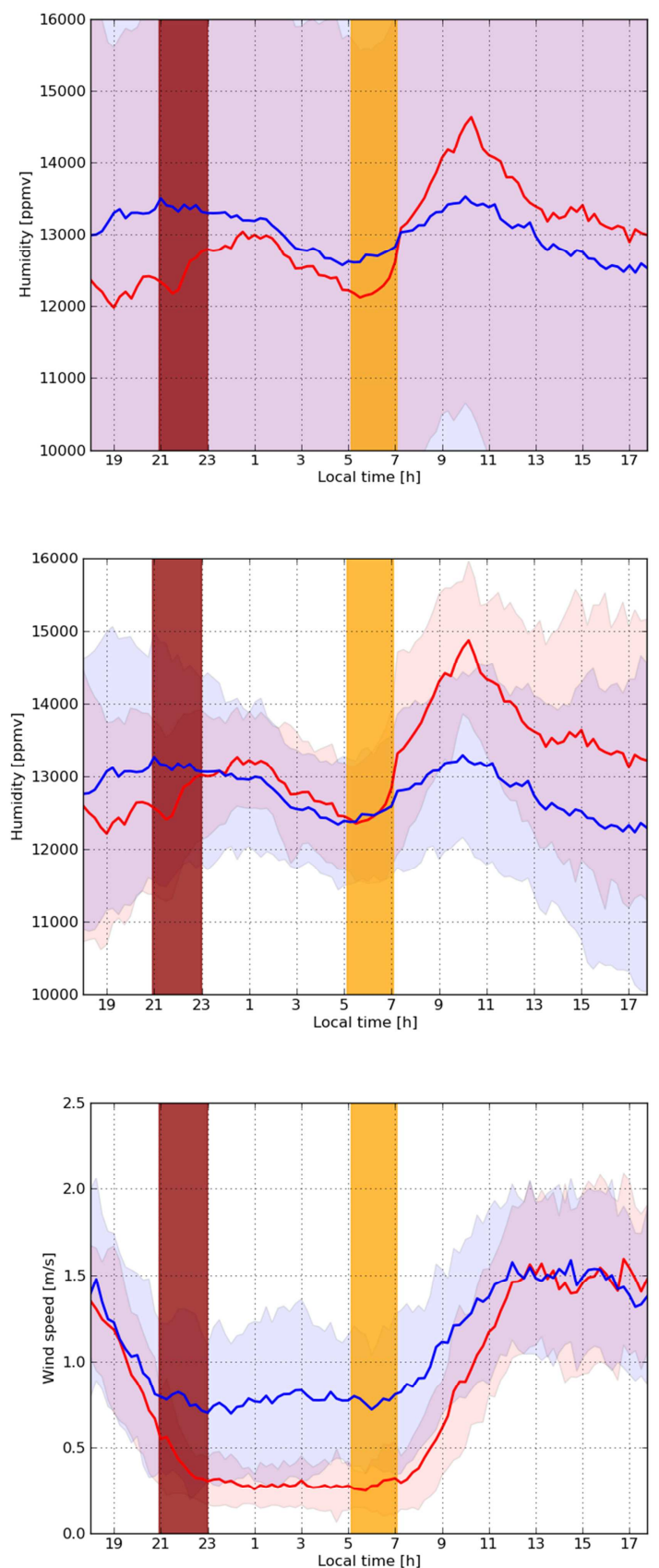

Figure S3. Diurnal cycles and two dominant clusters for (a) d-excess, (b) humidity, (c) humidity with subtracted mean value and (d) wind speed. 
e)

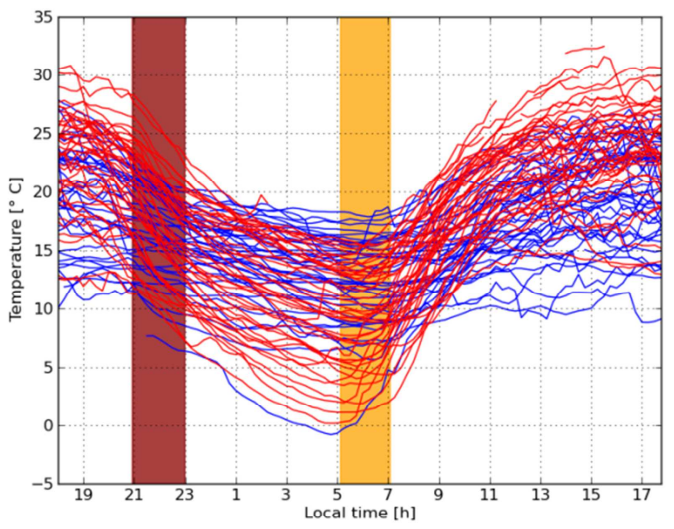

f)

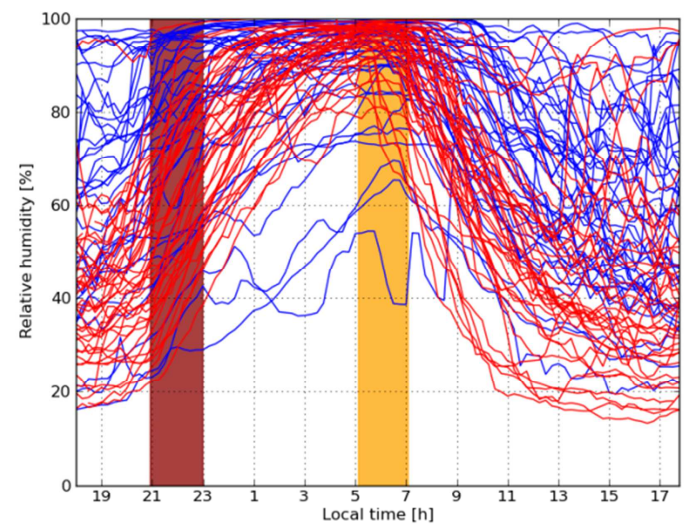

g)

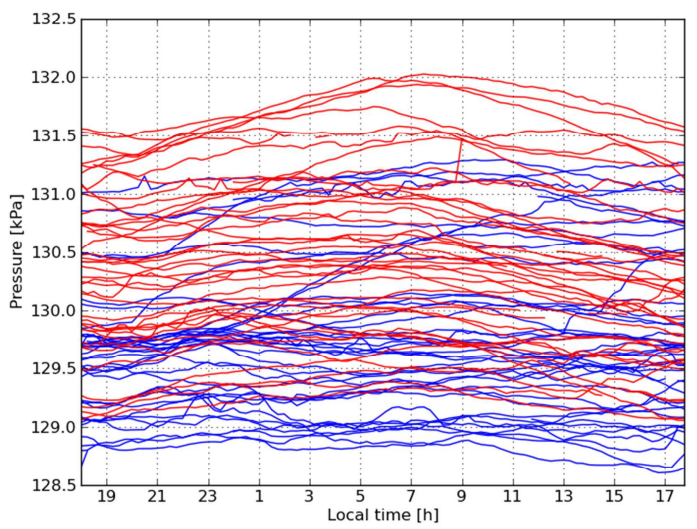

h)

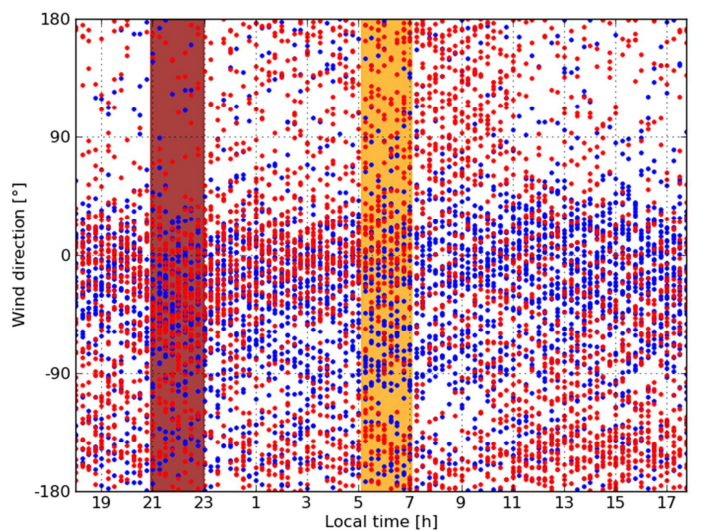

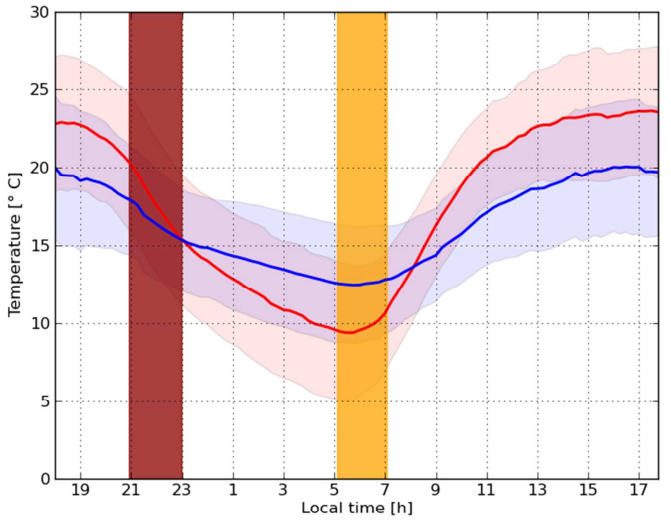
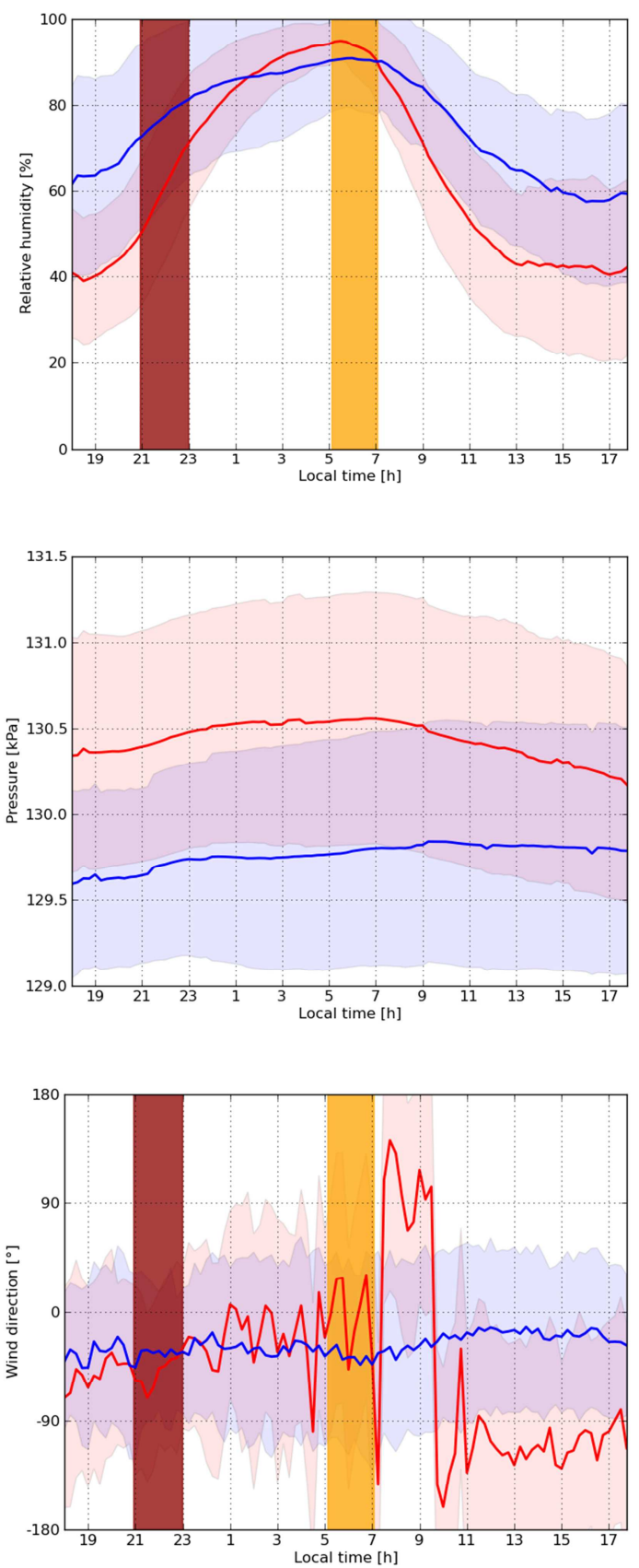

Figure S3 (continuation). Diurnal cycles and two dominant clusters for

(e) temperature, (f) relative humidity, (g) pressure and (h) wind direction. 